\title{
ARTICLE
}

Received 7 Jan 2015 | Accepted 1 Jun 2015 | Published 3 Jul 2015

\section{Intra-unit-cell magnetic correlations near optimal doping in $\mathrm{YBa}_{2} \mathrm{Cu}_{3} \mathrm{O}_{6.85}$}

\author{
L. Mangin-Thro' ${ }^{1}$ Y. Sidis ${ }^{1}$, A. Wildes ${ }^{2} \&$ P. Bourges ${ }^{1}$
}

The pseudo-gap phenomenon in copper oxide superconductors is central to any description of these materials as it prefigures the superconducting state itself. A magnetic intra-unit-cell order was found to occur just at the pseudo-gap temperature in four cuprate high- $T_{c}$ superconducting families. Here we present polarized neutron-scattering measurements of nearly optimally doped $\mathrm{YBa}_{2} \mathrm{Cu}_{3} \mathrm{O}_{6.85}$, carried out on two different spectrometers, that reveal several features. The intra-unit-cell order consists of finite-sized planar domains that are very weakly correlated along the $c$ axis. At high temperature, only the out-of-plane magnetic components correlate, indicating a strong Ising anisotropy. An aditional in-plane response develops at low temperature, giving rise to an apparent tilt of the magnetic moment. The discovery of these two regimes puts stringent constraints, which are tightly bound to the pseudo-gap physics, on the intrinsic nature of intra-unit-cell order.

\footnotetext{
${ }^{1}$ Laboratoire Léon Brillouin, IRAMIS/LLB, UMR12, CEA-CNRS, CEA-Saclay, Gif sur Yvette 91191, France. ${ }^{2}$ Institut Laue-Langevin, 71 avenue des martyrs, Grenoble 38000, France. Correspondence and requests for materials should be addressed to P.B. (email: philippe.bourges@cea.fr).
} 
U nderstanding high-temperature superconductivity requires a prior knowledge of the nature of the enigmatic pseudo-gap metallic state ${ }^{1}$, out of which the superconducting state condenses. In addition to the electronic orders involving charge degrees of freedom that were recently reported inside the pseudo-gap state ${ }^{2-4}$, polarized neutronscattering experiments reported the existence of a magnetic intra-unit-cell (IUC) order in four different cuprate families ${ }^{5-14}$. The IUC magnetic order breaks time-reversal symmetry but preserves the lattice translational symmetry (LTS). The IUC order develops below a temperature, $T_{\mathrm{mag}}$, that matches the pseudo-gap temperature, $T^{\star}$, as defined by resistivity measurements ${ }^{15,16}$, and where recent resonant ultrasound spectroscopy measurements in $\mathrm{YBa}_{2} \mathrm{Cu}_{3} \mathrm{O}_{6+x}$ (YBCO) show that the pseudo-gap state is a true symmetry breaking phase ${ }^{17}$. While bulk neutron-scattering measurements provide unambiguous evidence for an IUC magnetic order, such an order was not detected by local magnetic probe measurements $9,18,19$. The conundrum could be understood in terms of slowly fluctuating magnetic domains with finite $\operatorname{size}^{20}$, although their existence has not yet been established.

The observed IUC magnetism can be described simply as a $q=0$ antiferromagnetic state as it keeps the LTS, but requires an internal staggered magnetic pattern inside each unit cell. Such a state is qualitatively consistent with the loop current (LC) model for the pseudo-gap ${ }^{20,21}$. While out-of-plane magnetic moments should be produced by the planar confinement of LC, the observed magnetic moments also display an unexpected in-plane component, giving rise to a tilt of $\sim 45^{\circ}$ with respect to $c$ axis $^{5,9,11,12}$ at low temperature. Several attempts to explain this observation were proposed: an additional spin response through spin-orbit coupling ${ }^{22}$; a geometrical tilt of moment through delocalization of the $\mathrm{LC}$ over $\mathrm{CuO}$ octahedra or pyramids ${ }^{23,24}$; and a superposition of LC states through quantum effects ${ }^{25}$. Beside the LC model, electronic phases that break time-reversal symmetry, but preserve LTS, were proposed to pre-empt either a pair density wave state ${ }^{26}$ or a composite charge density wave (CDW) state ${ }^{27}$. Alternative scenarios consider an IUC order based on a nematic-like state with either $\operatorname{spin}^{5}$ or orbital ${ }^{28}$ moments on oxygens, or entangled spin and orbital degrees of freedom within an arrangement of magneto-electric multipoles ${ }^{29}$.

More experimental information is required on the size and lifetime of the magnetic domains and exact orientation of the magnetic moment to make progress on the understanding of the intrinsic nature of the IUC magnetic order and its interplay with the pseudo-gap physics. This can be achieved from studying compositions around optimal doping. Indeed, a fast decay of the magnetic intensity was reported from underdoped to optimally doped samples of $\mathrm{Bi}_{2} \mathrm{Sr}_{2} \mathrm{CaCu}_{2} \mathrm{O}_{8+\delta}$ (ref. 14). The magnetic intensity is proportional to $T_{\mathrm{mag}}$ in the underdoped regime, whereas this scaling suddenly breaks down on approaching optimal doping. These effects could actually be triggered by a redistribution of the magnetic scattering in momentum space, namely, a shortening of the magnetic correlation length on increasing hole doping.

We here report polarized neutron measurements on a $\mathrm{YBa}_{2} \mathrm{Cu}_{3} \mathrm{O}_{6.85}\left(T_{\mathrm{c}}=89 \mathrm{~K}\right)$ twinned single crystal, previously used to study the spin dynamics ${ }^{30,31}$, extending the study of the IUC order at a higher hole doping $p=0.15$ near optimal doping. We find short-range IUC correlations with finite-sized planar domains of $\sim \xi_{a} \sim 75 \AA$ at $100 \mathrm{~K}$, which are very weakly correlated along the $c$ axis. The magnetic intensity appears at high temperature with a net increase below $T_{\mathrm{mag}} \sim 200 \mathrm{~K}$. Polarization analysis reveals a strong Ising anisotropy at high temperature as originally predicted in the $\mathrm{LC}$ model ${ }^{21}$. Below $T_{\mathrm{mag}}$, transverse components correlate as well, leading to an apparent tilt of the magnetic moment at lower temperature. These different temperature dependencies of both in-plane and out-ofplane components of the magnetic moment suggest a distinct origin of each component, providing information on the nature of the tilt.

\section{Results}

IUC magnetism near optimal doping. At lower doping, evidence has been found for IUC magnetic order in YBCO by plotting normalized magnetic intensity, that is, the ratio of the spin flip (SF) to the non-SF (NSF) intensities at various Bragg reflections $s^{5,11,13}$. The IUC magnetic intensity occurs for Bragg reflections such as $\mathbf{Q}=(1,0, L)$ with $L=$ integer, whereas it is absent for $\mathbf{Q}=(0,0, L)$ or $\mathbf{Q}=(2,0, L)$ (refs 9,10). We show in Fig. 1 the results from the spectrometer $4 \mathrm{~F} 1$ (see Methods section) as a function of the temperature, for a polarization $\mathbf{P} \| \mathbf{Q}$, where the magnetic scattering is exclusively $\mathrm{SF}^{9}$. The raw data are shown in Supplementary Fig. 1 and the detailed analysis is given in Supplementary Note 1 following a method previously discussed $^{13,14}$. In Fig. 1a,b, we plot the normalized magnetic intensity obtained at $\mathbf{Q}=(2,0,0)$ and $\mathbf{Q}=(0,0,4)$, where a magnetic signal (if any) is beyond the threshold of detection $\left(<5 \times 10^{-5}\right)$. This serves as a reference calibration for the neutron flipping ratio.

Figure 1c shows the normalized magnetic intensity for the Bragg reflections $\mathbf{Q}=(1,0,1)$. Starting from zero scattering at high temperature, a magnetic signal appears below $T_{\text {mag }} \sim 200 \pm 20 \mathrm{~K}$. Our measurements first made on the Bragg reflection $\mathbf{Q}=(1,0,1)$ are confirmed by those on $\mathbf{Q}=(1,0,0)$ (Fig. 1d), where the data are further compared with a previous study on $\mathrm{YBa}_{2} \mathrm{Cu}_{3} \mathrm{O}_{6.6}$ (ref. 11). Such a value for $T_{\text {mag }}$ agrees with the recent accurate determination of $T^{*}$ from resistivity ${ }^{32}$. The magnetic signal displays a characteristic $T$ dependence that can be fitted by $\left(1-T / T_{\text {mag }}\right)^{2 \beta}$ with $2 \beta=0.4 \pm 0.17$, a value similar to what was found in $\mathrm{YBa}_{2} \mathrm{Cu}_{3} \mathrm{O}_{6.6}$ (ref. 11). Next, one can convert this magnetic amplitude in absolute units following a method described in previous studies ${ }^{5,9}$. The amplitude of the magnetic intensity, $I_{\text {mag, }}$ for both $\mathbf{Q}=(1,0, L)$ is $\sim 3 \times 10^{-4}$ of the nuclear intensity, giving $I_{\text {mag }} \sim 1.2$ mbarns per formula unit for $\mathbf{Q}=(1,0,0)$ and $I_{\mathrm{mag}} \sim 0.4$ mbarns per formula unit for $\mathbf{Q}=(1,0,1)$.

Short-range magnetic order. To gain a new insight of the IUC magnetism, we next carried out a polarized neutron experiment using the multidetector diffractometer D7 covering a wide range of scattering angle (see Methods section and Supplementary Note 2). A trajectory in the scattering plane (as shown in Fig. 2a) is simultaneously measured for a given sample rocking angle. A survey of a few trajectories in the $\mathbf{Q}=(H, 0, L)$ scattering plane showed the presence of magnetic scattering around $H=1$ not only for the Bragg reflection $(1,0,0)$ but also for non-integer $L$. In particular, the trajectory across $\mathbf{Q}=(1,0,0.25)$ (Fig. 2a) showed that the magnetic intensity is still large, whereas the nuclear Bragg scattering is significantly reduced (by about one order of magnitude), improving noticeably the ability to observe the magnetic scattering compared with the Bragg position at $\mathbf{Q}=(1,0,0)$. The magnetic scattering is thus short range along a $\operatorname{rod} \mathbf{Q}=(1,0, L)$ with an extension along $c^{\star}$, in contrast to more underdoped YBCO samples ${ }^{11}$. Similar short-range magnetic fluctuations around $H=1$ were also reported in $\mathrm{La}_{1.92} \mathrm{Sr}_{0.08} \mathrm{CuO}_{4}$ (ref. 7). One can therefore extract the in-plane correlations length $\xi_{a}$ (which is equivalent to $\xi_{b}$ as our sample is twinned) as well as the correlations length along $c^{*}$.

In Fig. $2 \mathrm{~b}, \mathrm{c}$, measurements along $\mathbf{Q}=(H, 0,0.25)$ showed that the magnetic signal increases on cooling. At high temperature $(300 \mathrm{~K})$, the magnetic signal is assumed to be featureless, whereas 

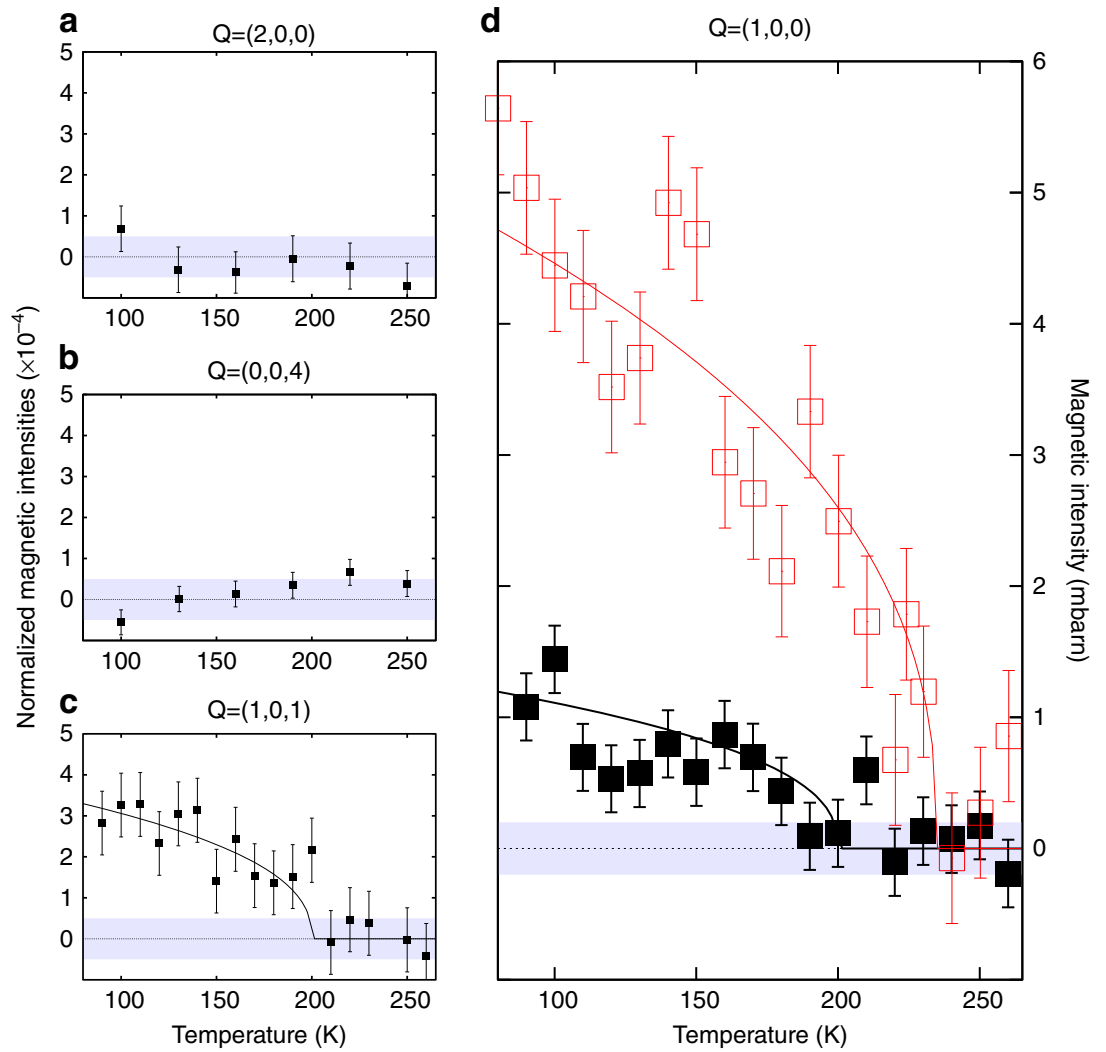

Figure 1 | Magnetic intensity at Bragg peak positions. 4F1 data: temperature dependencies of normalized magnetic intensity $\left(=I_{\text {mag }} / I_{\mathrm{NSF}}\right.$ where $I_{\mathrm{mag}}$ is the expected magnetic intensity of the IUC order) measured at the wave vector $(\mathbf{a}) \mathbf{Q}=(2,0,0)$ and $(\mathbf{b}) \mathbf{Q}=(0,0,4)$ as references, and at $(\mathbf{c}, \mathbf{d}) \mathbf{Q}=(1,0, L)$ for the IUC magnetic order study in the $\mathbf{P} \| \mathbf{Q}$ configuration. (c) $L=1$, (d) $L=0$. The data for $\mathbf{Q}=(1,0,0)\left(\mathrm{YBa}_{2} \mathrm{Cu}_{3} \mathrm{O}_{6.85}\right.$, full black squares) have been calibrated in absolute units using the intensity of the Bragg peak $\mathbf{Q}=(0,0,4)$ (ref. 5), and further compared with the $\mathrm{YBa}_{2} \mathrm{Cu}_{3} \mathrm{O}_{6.6}$ study $($ red empty squares $)^{11}$. Intensity error bars are statistical error bars calculated by the square root of the measured intensity.

at low temperature $(100 \mathrm{~K})$ there is a net enhancement of the magnetic intensity centred at $H=1$. From the $H$-dependences of Fig. 2c, one can extract the $q$-width by fitting a Gaussian, $\Delta H=0.025 \pm 0.004$ r.l.u. (reduced lattice units) (full-width at half-maximum). We observed no noticeable evolution of that $q$-width with temperature. Actually, $\Delta H$ is slightly broader than the resolution $\Delta H_{\text {res }}=0.0195 \pm 0.001$ r.l.u., suggesting shortrange correlations. One can extract a finite planar magnetic correlation length, $\xi_{a}=a /\left(\pi . \Delta H_{i}\right)$, where $a=3.85 \AA$ is the in-plane lattice parameter and $\Delta H_{i}=\sqrt{\Delta H^{2}-\Delta H_{\text {res }}^{2}}$ is obtained by deconvolution from the resolution: $\xi_{a} \sim 20 a \sim 75 \AA$. Such a correlation length cannot be determined on $4 \mathrm{~F} 1$ due to its poorer $H$-resolution (see Methods section).

Next, to improve the statistics of signal, we proceeded to a partial integration of the scattering intensities over 10 detectors (Supplementary Note 3). This averaged intensity, $I_{\mathrm{av}}$, is reported as a function of temperature (Fig. 3a) along the magnetic rod $\mathbf{Q}=(1,0, L)$ for two $L$ values $(L=0.25$ and 0.5$)$ off the Bragg position. Here we have summed up the cross-sections of the three different neutron polarizations in the SF channel $\overline{\mathrm{I}}_{X}^{\mathrm{SP}}+\overline{\mathrm{I}}_{Y}^{\mathrm{SF}}+\overline{\mathrm{I}}_{Z}^{\mathrm{SF}}$. Using that method, weak magnetic correlation can be detected even at high temperature above $T_{\mathrm{mag}} \sim 200 \mathrm{~K}$. As the sample is cooled, the comparison of the magnetic intensity at both $L$ values in Fig. 3a shows that the magnetic intensities at first grow similarly at both $L$ and then more rapidly for $L=0.25$. That behaviour suggests a gradual redistribution of the magnetic intensity along the $(1,0, L)$ rod towards the magnetic Bragg positions, pointing towards a two-dimensional (2D) to threedimensional (3D) crossover of the magnetic correlations with temperature.
By converting the magnetic intensity to absolute units, one can compare data from both $4 \mathrm{~F} 1$ and D7 (Fig. 3b): the scattering is broader along $c^{*}$ than the $L$-resolution width given by $\Delta L_{\text {res }}=0.2$ r.l.u. (see Methods section). Using a Gaussian fit, one estimates the full-width at half-maximum of the magnetic signal along (001), $\Delta L=0.65 \pm 0.05$ r.l.u. at $100 \mathrm{~K}$, out of which one can extract the correlation length along $c^{\star}, \xi_{c}=c /\left(\pi \cdot \Delta L_{i}\right)$, where $\Delta L_{i}=\sqrt{\Delta L^{2}-\Delta L_{\text {res }}^{2}}$ is obtained by deconvolution from the resolution. It gives a very short correlation length along $c^{*}, \xi_{c}$ $\sim 0.5 c$ at $T=100 \mathrm{~K}$. That observed $L$-dependence is intermediate between two limiting cases: 2D correlations (dotted line in Fig. 3b) as it is observed in $\mathrm{La}_{1.92} \mathrm{Sr}_{0.08} \mathrm{CuO}_{4}$ (ref. 7) and well-defined 3D ordering (shaded Gaussian peaks in Fig. 3b) in more underdoped YBCO where $\xi_{c}>7 c$ (refs 9,11).

Magnetic components. One can also use the temperature dependence of the averaged intensity to estimate the directional components of the magnetic moment, $M=\left(M_{\mathrm{a}}, M_{\mathrm{b}}, M_{\mathrm{c}}\right)$. For a twinned sample, both directions $(1,0)$ and $(0,1)$ are equivalent, and one can define $M_{\mathrm{ab}}^{2}=M_{\mathrm{a}}^{2}+M_{\mathrm{b}}^{2}$ as the in-plane component and $M_{\mathrm{c}}^{2}$ as the out-of-plane component ${ }^{9}$. As described in the Supplementary Note 4 , the cross-section in the SF channel $\overline{\mathrm{I}}_{Z}^{\mathrm{SF}}$ essentially corresponds to the out-of-plane magnetic component, $M_{\mathrm{c}}^{2}$, in the limit $q_{1}=(2 \pi L / c) /|\mathrm{Q}| \rightarrow 0$ (as it is the case for $L=0.25)$, and conversely the quantity $\overline{\mathrm{I}}_{X}^{\mathrm{SF}}+\overline{\mathrm{I}}_{Y}^{\mathrm{SP}}-\overline{\mathrm{I}}_{Z}^{\mathrm{SF}}$ directly probes the in-plane component $M_{\mathrm{ab}}^{2}$. In Fig. 3c,d, we report the temperature evolution of the out-of-plane component $M_{\mathrm{c}}^{2}$ and the in-plane component $M_{\mathrm{ab}}^{2}$. Interestingly, both cross-sections exhibit noticeably distinct temperature dependences. At high 


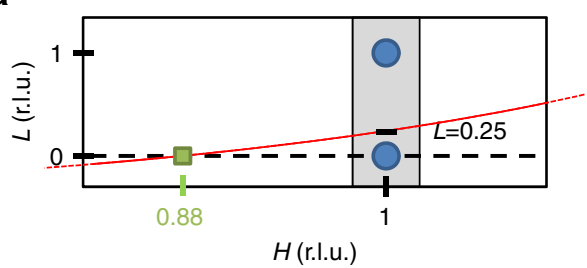

b

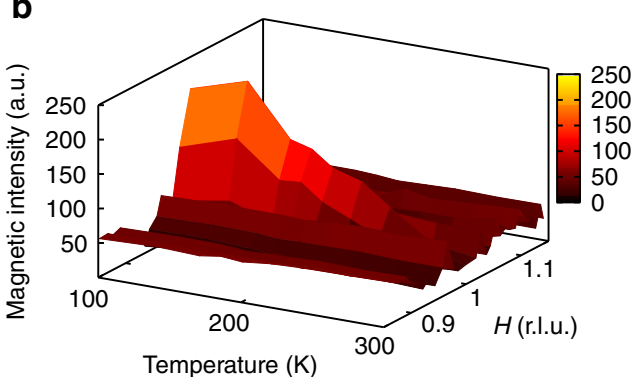

C

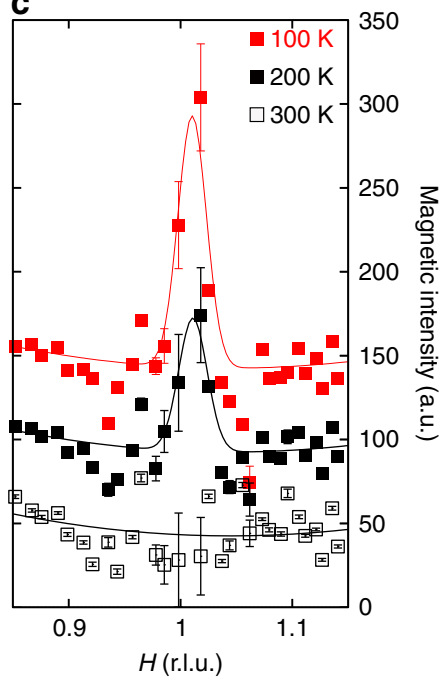

Figure 2 | In-plane momentum dependence of the magnetic correlations. (a) Schematic view of the $(H, O, L)$ reciprocal space probed on D7 with the measured trajectory going through $(1,0,0.25)$ in red. The grey area represents the location of the magnetic intensity. r.l.u., reduced lattice units. The green spot represents the location where one observes diffuse scattering around $H=0.88$ shown in Fig. 4. (b) 3D plot of the magnetic intensity in the SF channel around $\mathbf{Q}=(1,0,0.25)$ as a function of temperature and wave vector. (c) Cuts of the $3 \mathrm{D}$ map along $(H, 0,0.25)$ at three temperatures: $100 \mathrm{~K}$ (red squares), $200 \mathrm{~K}$ (brown squares) and $300 \mathrm{~K}$ (black empty squares). Data obtained in channels $X$ and $Z$ are here averaged. Intensity error bars are statistical error bars calculated by the square root of the measured intensity.

a

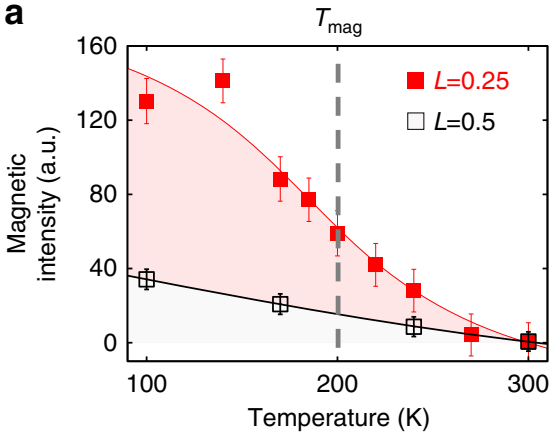

b

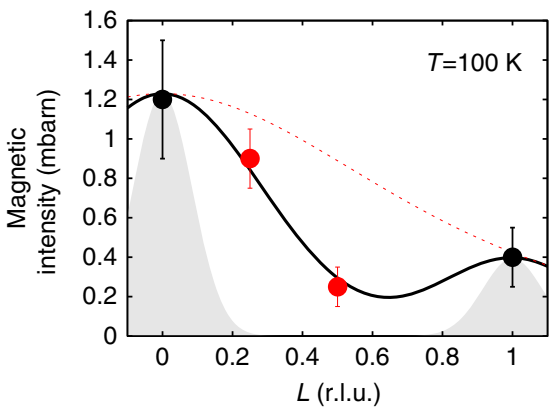

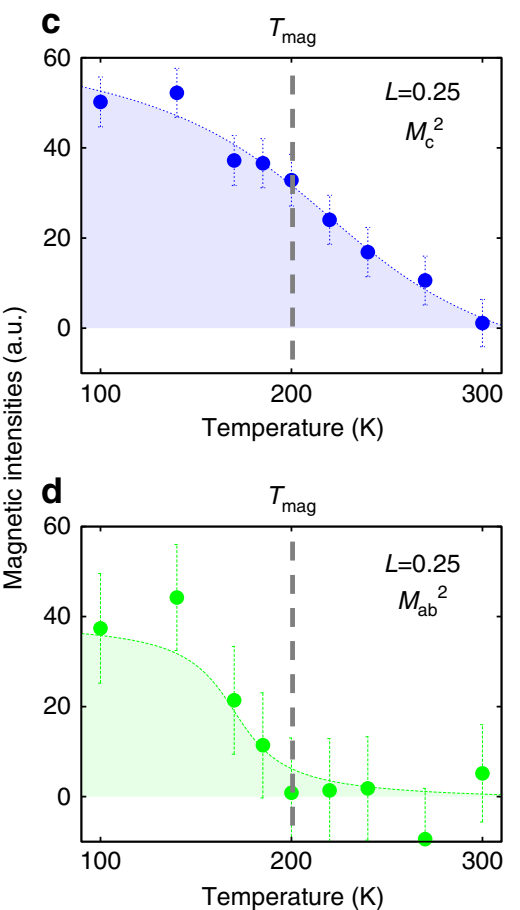

Figure 3 | Temperature and out-of-plane momentum dependence of the magnetic correlations. (a) Temperature dependences of the magnetic intensity, $\overline{\mathrm{I}}_{X}^{\mathrm{SF}}+\overline{\mathrm{I}}_{Y}^{\mathrm{SF}}+\overline{\mathrm{I}}_{Z}^{\mathrm{SF}}$ (background subtracted) for two $L$. (b) Magnetic intensity in absolute units from $4 \mathrm{~F} 1$ (black symbols) and D7 (red symbols) versus $L$ at $100 \mathrm{~K}$. The dashed areas represent Bragg peaks with the L-resolution of the spectrometers. The red dotted line corresponds to the universal decay of the magnetic intensity along the magnetic rod $\mathbf{Q}=(1,0, L)$ found in all cuprates ${ }^{8,14}$. Temperature dependence of the magnetic components for $L=0.25$ : (c) the out-ofplane component $M_{c}^{2}$ and (d) the in-plane component $M_{a b}^{2}$ (Supplementary Note 4). Solid lines are guides to the eye. Intensity error bars are statistical error bars calculated by the square root of the measured intensity.

temperature, the in-plane component vanishes, meaning that the correlated magnetic fluctuations $\left\langle M^{2}\right\rangle$ essentially correspond to the out-of-plane component. That strong Ising character (along $c^{\star}$ ) remains down to $T_{\text {mag. }}$ Below $T_{\text {mag, }}$ the in-plane component also increases. As $I_{\mathrm{av}} \propto I_{0} / \xi_{a}$ (where $I_{0}$ is the magnetic peak intensity), it indicates that both magnetic components have a distinct temperature-dependent evolution in their amplitudes and/or in their correlation lengths.

Following the definition of the magnetic moment, the ratio between $M_{\mathrm{ab}}$ and $M_{\mathrm{c}}$ at $100 \mathrm{~K}$ can be used to define an apparent 

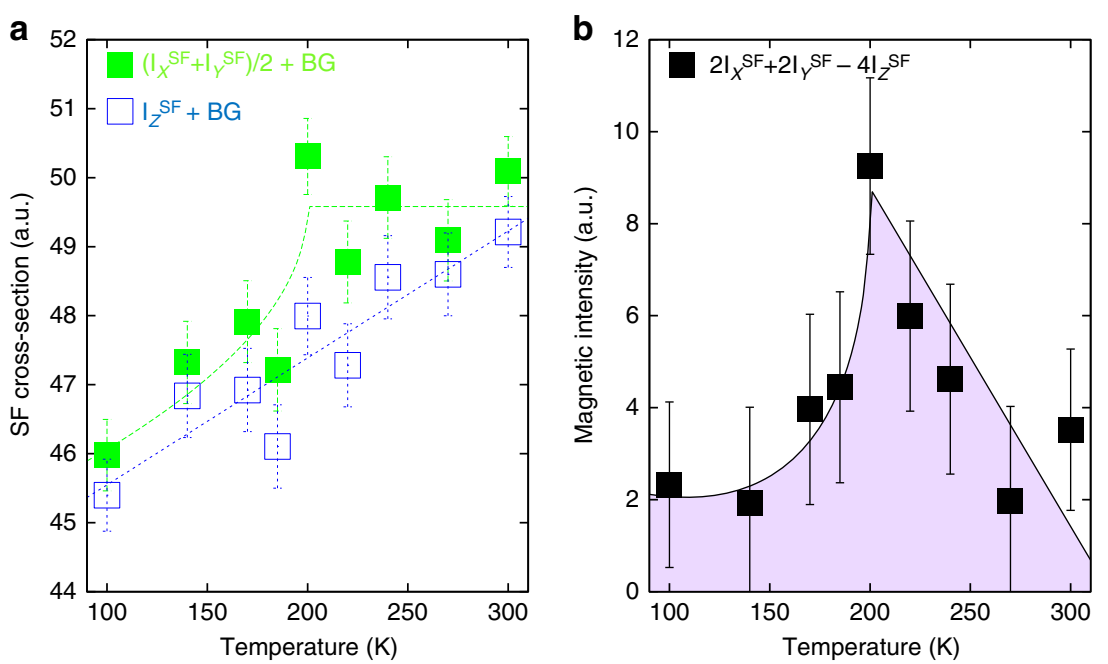

Figure 4 | Temperature dependence of the magnetic diffuse scattering. (a) Temperature dependencies of the $S F$ cross-sections around $\mathbf{Q}$ for $H \simeq 0.88$ and $L=0$ (at the green spot of the Fig. 2a) for two polarizations: $\left(\frac{\mathrm{d} \sigma}{\mathrm{d} \Omega}\right)_{\mathrm{SF}}^{Z}=\mathrm{I}_{Z}^{\mathrm{SF}}+\mathrm{BG}$ (open symbols) and $\left[\left(\frac{\mathrm{d} \sigma}{\mathrm{d} \Omega}\right)_{\mathrm{SF}}^{X}+\left(\frac{\mathrm{d} \sigma}{\mathrm{d} \Omega}\right)_{\mathrm{SF}}^{Y}\right] / 2=\left(\mathrm{I}_{X}^{\mathrm{SF}}+\mathrm{I}_{Y}^{\mathrm{SF}}\right) / 2+\mathrm{BG}(\mathrm{full}$ symbols). BG represents a spin incoherent background scattering that weakly decreases with temperature ${ }^{33}$. (b) Differential cross-sections $S_{\text {mag }}=\left(2 I_{X}^{S F}+2 I_{Y}^{S F}-4 I_{Z}^{S F}\right)$ obtained from the polarization analysis around $(0.88,0,0)$. Solid lines are guides to the eye. Intensity error bars are statistical error bars calculated by the square root of the measured intensity.

tilt angle, $\theta$, of the magnetic moment with respect to the $c^{*}$ axis as $\tan (\theta)=M_{\mathrm{ab}} / M_{\mathrm{c}}$ (ref. 9) from Fig. 3c,d, $\tan (\theta) \sim 0.84$, giving $\theta \sim 40^{\circ} \pm 9^{\circ}$ for $L=0.25$. As shown by Fig. $3 \mathrm{~b}$, the magnetic correlations at $L=0.25$ belong to the same peak as $L=0$, so that tilt value holds for $L=0$ as well. Actually, a similar value for the tilt for measurements at $L=1$ has been reported previously for more underdoped YBCO samples ${ }^{5,9,11}$.

Further, one observes a diffuse magnetic scattering at wave vectors away from the magnetic rod in addition to the correlated magnetic intensity occuring along $(1,0, L)$. That diffuse scattering is at least one order of magnitude weaker. Figure 4a shows the SF intensities for two polarizations around $H=0.88$ and $L=0$ (see Fig. $2 c$ for the momentum location). On cooling, these diffuse SF intensities decrease. This is consistent with a shift of magnetic scattering towards $H=1$ (Fig. 3a), likely owing to a smooth increase of the magnetic correlation length on cooling.

We applied the standard D7 polarization analysis relation for paramagnetic systems, typically valid for disordered magnetism ${ }^{33}$ to get the differential cross-section, $S_{\mathrm{mag}}=\left(2 \mathrm{I}_{X}^{\mathrm{SF}}+2 \mathrm{I}_{Y}^{\mathrm{SF}}-4 \mathrm{I}_{Z}^{\mathrm{SF}}\right)$ from these diffuse SF intensities. That quantity, $S_{\text {mag, }}$, does not depend on the background level, assuming that the background is independant of the polarization. Interestingly, $S_{\text {mag }}$ exhibits a net maximum as a function of temperature at $T_{\text {mag }}=200 \mathrm{~K}$ (Fig. $4 \mathrm{~b}$ ). Such a cusp shape is generally characteristic of critical-like scattering around an ordering temperature, although here this does not apply fully as the correlations lengths remain finite. Acordingly, at wave vector $(H, 0,0)$ far away from $H=1$ (say $H<0.8$ ), the cusp shape disappears. Looking back to the SF intensities, the cusp can be nearly seen in the $(X+Y) / 2$ channel although it does not occur for the $Z$ polarization (Fig. 4a). The fact that the cusp is more prominent in $S_{\text {mag }}$ underlines different thermal dependencies of the magnetic components of the fluctuating moments (Supplementary Note 5).

\section{Discussion}

IUC magnetic correlations are still sizeable around optimal doping below $T_{\text {mag }} \sim 200 \mathrm{~K}$ in $\mathrm{YBa}_{2} \mathrm{Cu}_{3} \mathrm{O}_{6.85} \quad\left(T_{\mathrm{c}}=89 \mathrm{~K}\right.$, $p=0.15)$. Compared with samples with a lower hole doping level, the measured intensity at the Bragg position is nevertheless strongly reduced. More precisely, the magnetic intensity in $\mathrm{YBa}_{2} \mathrm{Cu}_{3} \mathrm{O}_{6.85}$ is $\sim 4$ times weaker (Fig. 1d) than previous measurements performed on $\mathrm{YBa}_{2} \mathrm{Cu}_{3} \mathrm{O}_{6.6}$ at $\mathbf{Q}=(1,0,0)$ (ref. 11) and $\mathbf{Q}=(1,0,1)$ (ref. 5). Following the same hypotheses as before to determine the magnetic moment ${ }^{9}$ and keeping in mind that the magnetic intensity is proportional to $M^{2}$, the magnetic moment would then be estimated to be only $\sim 0.05 \mu_{\mathrm{B}}$ compared with $0.1 \mu_{B}$ for more underdoped $\mathrm{YBCO}^{5,9}$. In $\mathrm{Bi}_{2} \mathrm{Sr}_{2} \mathrm{CaCu}_{2} \mathrm{O}_{8+\delta}$ compounds, the magnetic intensity similarly decreases rapidly from the underdoped to optimally doped samples ${ }^{14}$. In this study, we have established that the magnetic intensity is proportional to $T_{\text {mag }}$ in the underdoped regime, whereas this scaling suddenly breaks down on approaching optimal doping.

We suggested that this effect could be triggered by a redistribution of the magnetic scattering in momentum space, namely, a shortening of the magnetic correlation length on increasing hole doping. As one observes short-range magnetic correlations in $\mathrm{YBa}_{2} \mathrm{Cu}_{3} \mathrm{O}_{6.85}$, one can test that scenario here considering the observed correlation lengths, $\xi_{a, c}$, at $100 \mathrm{~K}$. To perform that comparison, one needs to compare $\xi_{a, c}$ with the resolution fonction of the 4F1 spectrometer where we have measured both YBCO samples. With the broad resolution along $H$ of the spectrometer $4 \mathrm{~F} 1$ (see Methods), the observed in-plane correlations length, $\xi_{a} \sim 20 a$, is too large to broaden the in-plane $H$-width and does not affect the peak intensity. In contrast, the large $L$-width along $c^{\star}, \Delta L=0.65 \pm 0.05$ r.l.u., is $\sim 3$ times broader than the resolution peak width along $c^{\star}$. At lower doping, the peak was found to be resolution limited along $c^{\star}$ in $\mathrm{YBa}_{2} \mathrm{Cu}_{3} \mathrm{O}_{6.6}$ (ref. 11). Therefore, the $L$-integrated intensity is consistent with the ratio of $T_{\text {mag }}$ measured for the two different dopings. The broad $L$-dependence can explain the sharp decrease of the peaked magnetic intensity versus doping ${ }^{14}$.

The observation of short-range magnetic correlations may also explain why the observation of the IUC magnetic order has not yet been corroborated by magnetic local probe measurements ${ }^{9}$, such as nuclear magnetic resonance ${ }^{18,19}$, owing to their much longer timescales. Indeed, finite magnetic correlation lengths could be bound to the existence of slowly fluctuating domains, with a characteristic timescale proportional to the square of the correlation length ${ }^{20}$. In such a line of thought, the correlation 
length ought to stay finite even at lower doping to explain the absence of a magnetic signal in local probes. However, one cannot give a value of the timescale from our measurement. It can only be longer than our neutron resolution timescale $\left(\sim 10^{-11}\right.$ s; ref. 5$)$.

Our results show finite magnetic correlation lengths, this can be due to disorder in our sample. It is important to remember that the IUC order is stable in the presence of disorder as magnetic intensity at the Bragg peak position has been found near optimal doping in $\mathrm{Bi}_{2} \mathrm{Sr}_{2} \mathrm{CaCu}_{2} \mathrm{O}_{8+\delta}$ (ref. 8), where scanneling tunnel microscopy studies ${ }^{34}$ report a significant disorder related to oxygen. Chain fragments in the $\mathrm{CuO}$ chains of YBCO can induce such a disorder in the neighbouring $\mathrm{CuO}_{2}$ planes that could play a role in the proliferation of magnetic domains. Alternatively, one cannot exclude that the domain formation could also be the result of a competition with another electronic instability.

In terms of the phase diagram, it is also worth pointing out that $\mathrm{X}$-ray measurements recently revealed CDW order $^{2,35}$, which appears systematically at lower temperature $T_{\mathrm{CDW}}<T_{\mathrm{mag}}$ for all doping and also for different cuprates ${ }^{36}$. Further, the correlation lengths of the CDW state in $\mathrm{YBa}_{2} \mathrm{Cu}_{3} \mathrm{O}_{6.85}$ is $\xi_{\mathrm{CDW}} \sim 8 a<\xi_{a}$ $\sim 20 a$ weaker than the correlation lengths of the IUC order. That suggests the pre-eminence of the IUC order over the CDW instability. Disorder has been invoked also to explain the shortrange CDW correlations ${ }^{19}$.

We report the first signature of a decoupling of the magnetic moment components as they exhibit noticeably distinct temperature dependences. The component along $c^{\star}$ correlates first at high temperature, whereas the in-plane component is absent. Interestingly, that reveals a strong Ising character as it is expected in the original LC model ${ }^{9,21}$. In contrast, the correlated in-plane component develops only below $T_{\text {mag }}$ giving rise to an apparent tilt of the magnetic moment. Most theories that are interpretating the origin of the tilt, $\theta$, focus on the Bragg peak $L=1$ (refs 24,28,29). Our results challenge them as it remains $\sim 40^{\circ}$ whatever the $L$ value. It actually does not favour models in which $\theta$ goes to zero at $L=0$, such as LC models that include apical oxygens as an explanation for the tilt ${ }^{23,24}$, where one would expect a L-dependent tilt. More generally, the observed temperature dependence of $\theta$ is not consistent with theories where the tilt direction is due to rigid geometrical factors. The same conclusion holds when the in-plane component is related to spin orbit ${ }^{22}$. In contrast, in the LC approach ${ }^{25}$, where the planar magnetic component arises from quantum superposition of LC patterns, an Ising-like response could be recovered at high temperature once thermal fluctuations would overcome quantum effects.

Our study highlights the existence of short-range IUC magnetic correlations at very high temperature with an Ising anisotropy consistent with orbital magnetism produced by LCs. Meanwhile, the mysterious in-plane IUC correlations only develop around $T_{\text {mag }}$, which matches the pseudo-gap temperature. Concommitently, the magnetic response undergoes a $2 \mathrm{D}$ to $3 \mathrm{D}$ crossover. Our study further suggests that it may exist a close connection between the pseudo-gap and the balance between inplane and out-of-plane IUC magnetic correlations.

\section{Methods}

Polarized neutron diffraction. The polarized neutron experiments have been performed on two spectrometers: the triple axis spectrometer 4F1 (Orphée, Saclay; Supplementary Note 1) and the multidetector diffractometer D7 (ILL, Grenoble; Supplementary Note 2). For both, a polarizing super-mirror (bender) and a Mezei flipper are inserted on the incoming neutron beam to select neutrons with a given spin. In addition, a filter (pyrolytic graphite for $4 \mathrm{~F} 1$ or beryllium for D7) is put before the bender to remove high harmonics. After the sample, the final polarization, $P$, is analysed by an Heusler analyser on $4 \mathrm{~F} 1$, whereas $\mathrm{D} 7$ is equipped with an array of polarizing benders in front of the detectors. For each wave vector $\mathbf{Q}$, the scattered intensity is measured in both SF and NSF channels. For the measurements on $4 \mathrm{~F} 1$, the incident and final neutron wave vector are set to $k_{\mathrm{I}}=k_{\mathrm{F}}=2.57 \AA^{-1}$. On the diffractometer D7, the incident wave vector is taken as $k_{\mathrm{I}}=1.29 \AA^{-1}$. As we are using cold neutrons, we had $\sim 4$ times better $H$-resolution compared with4F1, where $\Delta H_{\text {res }} \simeq 0.08$ r.l.u. with a similar $L$-resolution $\Delta L_{\text {res }} \sim 0.2$ r.l.u., as this quantity is mostly related to the sample mosaicity. Following previous studies $5,6,8,11,12,14$, the search for magnetic order in the pseudo-gap phase is performed around Bragg reflections $\mathbf{Q}=(1,0, L)$ with integer $L$ values. The sample was then aligned in the scattering plane $(1,0,0) /(0,0,1)$, thusonly wave vectors spanning the $(H, 0, L)$ plane are accessible. D7 is equipped with a fixed $X Y Z$ polarization mode ${ }^{33}$ with $Z$ being vertical and $X$ and $Y$ are pointing along arbitrary in-plane directions. For all of the three polarizations $X, Y$ and $Z$, the SF and NSF channels were systematically measured over a few rocking angles of the sample to map the magnetic scattering We then performed data reduction adapting the standard procedure ${ }^{33}$ and forced the magnetic scattering to be featureless at $300 \mathrm{~K}$ for any polarization direction. This was made to find evidence for small magnetic intensities. The procedure includes background subtraction, flipping ratio and vanadium corrections. Further, the conversion in absolute units has been performed using the Bragg peak $\mathbf{Q}=(1,0,0)$ intensity (see Supplementary Note 2 for details).

\section{References}

1. Norman, M. R. \& Pépin, C. The electronic nature of high temperature cuprate superconductors. Rep. Prog. Phys. 66, 1547-1610 (2003).

2. Keimer, B., Kivelson, S. A., Norman, M. R., Uchida, S. \& Zaanen, J. From quantum matter to high-temperature superconductivity in copper oxides. Nature 518, 179-186 (2015).

3. Davis, J. C. \& Lee, D.-H. Concepts relating magnetic interactions, intertwined electronic orders, and strongly correlated superconductivity. Proc. Natl Acad. Sci. USA 110, 17623-17630 (2013).

4. Efetov, K. B., Meier, H. \& Pépin, C. Pseudogap state near a quantum critical point. Nat. Phys. 9, 442-446 (2013).

5. Fauqué, B. et al. Magnetic order in the pseudogap phase of high-Tc superconductors. Phys. Rev. Lett. 96, 197001 (2006).

6. Li, Y. et al. Unusual magnetic order in the pseudogap region of the superconductor $\mathrm{HgBa}_{2} \mathrm{CuO}_{4+\delta}$. Nature 455, 372-375 (2008).

7. Balédent, V. et al. Two-dimensional orbital-like magnetic order in the hightemperature $\mathrm{La}_{2}-\mathrm{Sr}_{\mathrm{x}} \mathrm{CuO}_{4}$ superconductor. Phys. Rev. Lett. 105, 027004 (2010).

8. De Almeida-Didry, S. et al. Evidence for intra-unit-cell magnetic order in $\mathrm{Bi}_{2} \mathrm{Sr}_{2} \mathrm{CaCu}_{2} \mathrm{O}_{8+\delta}$. Phys. Rev. B 86, 020504 (2012).

9. Bourges, P. \& Sidis, Y. Novel magnetic order in the pseudogap state of high-Tc copper oxides superconductors. C. R. Physique 12, 461-479 (2011).

10. Sidis, Y. \& Bourges, P. Evidence for intra-unit-cell magnetic order in the pseudo-gap state of high-Tc cuprates. J. Phys. Conf. Ser. 449, 012012 (2013).

11. Mook, H. A., Sidis, Y., Fauqué, B., Balédent, V. \& Bourges, P. Observation of magnetic order in a superconducting $\mathrm{YBa}_{2} \mathrm{Cu}_{3} \mathrm{O}_{6.6}$ single crystal using polarized neutron scattering. Phys. Rev. B 78, 020506 (2008).

12. Li, Y. et al. Magnetic order in the pseudogap phase of $\mathrm{HgBa}_{2} \mathrm{CuO}_{4+\delta}$ studied by spin-polarized neutron diffraction. Phys. Rev. B 84, 224508 (2011).

13. Balédent, V. et al. Evidence for competing magnetic instabilities in underdoped $\mathrm{YBa}_{2} \mathrm{Cu}_{3} \mathrm{O}_{6+x}$. Phys. Rev. B 83, 104504 (2011).

14. Mangin-Thro, L. et al. Characterization of the intra-unit-cell magnetic order in $\mathrm{Bi}_{2} \mathrm{Sr}_{2} \mathrm{CaCu}_{2} \mathrm{O}_{8}+\delta$. Phys. Rev. B 89, 094523 (2014).

15. Ito, T., Takenaka, K. \& Uchida, S. Systematic deviation from T-linear behavior in the in-plane resistivity of $\mathrm{YBa}_{2} \mathrm{Cu}_{3} \mathrm{O}_{7-\delta}$ : evidence for dominant spin scattering. Phys. Rev. Lett. 70, 3995-3998 (1993).

16. Ando, Y., Komiya, S., Segawa, K., Ono, S. \& Kurita, Y. Electronic phase diagram of high-Tc cuprate superconductors from a mapping of the in-plane resistivity curvature. Phys. Rev. Lett. 93, 267001 (2004).

17. Shekhter, A. et al. Bounding the pseudogap with a line of phase transitions in $\mathrm{YBa}_{2} \mathrm{Cu}_{3} \mathrm{O}_{6+\delta}$. Nature 498, 75-77 (2013).

18. Mounce, A. M. et al. Absence of static orbital current magnetism at the apical oxygen site in $\mathrm{HgBa}_{2} \mathrm{CuO}_{4+\delta}$ from NMR. Phys. Rev. Lett. 111, 187003 (2013).

19. Wu, T. et al. Incipient charge order observed by NMR in the normal state of $\mathrm{YBa}_{2} \mathrm{Cu}_{3} \mathrm{O}_{y}$. Nat. Commun. 6, 6438 (2015).

20. Varma, C. M. Pseudogap in cuprates in the loop-current ordered state. J. Phys. Condens. Matter 26, 505701 (2014).

21. Varma, C. M. Theory of the pseudogap state of the cuprates. Phys. Rev. B 73, 155113 (2006).

22. Aji, V. \& Varma, C. M. Spin order accompanying loop-current order in cuprate superconductors. Phys. Rev. B 75, 224511 (2007).

23. Weber, C., Läuchli, A., Mila, F. \& Giamarchi, T. Orbital currents in extended Hubbard models of high-Tc cuprates. Phys. Rev. Lett 102, 017005 (2009).

24. Lederer, S. \& Kivelson, S. A. Observable NMR signal from circulating current order in YBCO. Phys. Rev. B 85, 155130 (2012). 
25. He, Y. \& Varma, C. M. Theory of polarized neutron scattering in the loopordered phase of cuprates. Phys. Rev. B 86, 035124 (2012).

26. Agterberg, D. F., Melchert, D. S. \& Kashyap, M. K. Emergent loop current order from pair density wave superconductivity. Phys. Rev. B 91, 054502 (2015).

27. Wang, Y. \& Chubukov, A. Charge order and loop currents in hole-doped cuprates. Phys. Rev. B 90, 035149 (2014).

28. Moskvin, A. S. Pseudogap phase in cuprates: oxygen orbital moments instead of circulating currents. JETP Lett. 96, 424-429 (2012).

29. Lovesey, S. W., Khalyavin, D. D. \& Staub, U. Ferro-type order of magneto-electric quadrupoles in the pseudo-gap phase of $\mathrm{YBa}_{2} \mathrm{Cu}_{2} \mathrm{O}_{6+x}$ unearthed by neutron diffraction. Preprint at http://arxiv.org/abs/1501.02427 (2015).

30. Bourges, P. et al. The spin excitation spectrum in superconducting $\mathrm{YBa}_{2} \mathrm{Cu}_{3} \mathrm{O}_{6.85}$. Science 288, 1234 (2000).

31. Pailhès, S. et al. Resonant magnetic excitations at high energy in superconducting $\mathrm{YBa}_{2} \mathrm{Cu}_{3} \mathrm{O}_{6.85}$. Phys. Rev. Lett. 93, 167001 (2004).

32. Alloul, H., Rullier-Albenque, F., Vignolle, B., Colson, D. \& Forget, A. Superconducting fluctuations, pseudogap and phase diagram in cuprates. Europhys. Lett. 91, 37005 (2010).

33. Stewart, J. R. et al. Disordered materials studied using neutron polarization analysis on the multi-detector spectrometer, D7. J. Appl. Cryst. 42, 69-84 (2009).

34. McElroy, K. et al. Atomic-scale sources and mechanism of nanoscale electronic disorder in $\mathrm{Bi}_{2} \mathrm{Sr}_{2} \mathrm{CaCu}_{2} \mathrm{O}_{8+\delta}$. Science 309, 1048 (2005).

35. Blanco-Canosa, S. et al. Resonant X-ray scattering study of charge-density wave correlations in $\mathrm{YBa}_{2} \mathrm{Cu}_{3} \mathrm{O}_{6+x}$. Phys. Rev. B 90, 054513 (2014).

36. Tabis, W. et al. Connection between charge-density-wave order and charge transport in the cuprate superconductors. Nat. Commun. 5, 5875 (2014).

\section{Acknowledgements}

We thank C.M. Varma, M.K. Chan and M. Greven for fruitful discussions. We also acknowledge financial support from the grant EXCELCIUS of the Labex PALM of the Universite Paris-Saclay and the project UNESCOS (contract ANR-14-CE05-0007) of the ANR.

\section{Author contributions}

L.M.-T., Y.S. and P.B. performed the experiment at LLB Saclay; L.M.-T., A.W. and P.B. performed the experiment at ILL Grenoble; L.M.-T. and P.B. analysed all the neutron data; all authors wrote the manuscript; and P.B. supervised the project.

\section{Additional information}

Supplementary Information accompanies this paper at http://www.nature.com/ naturecommunications

Competing financial interests: The authors declare no competing financial interests.

Reprints and permission information is available online at http://npg.nature.com/ reprintsandpermissions/

How to cite this article: Mangin-Thro, L. et al. Intra-unit-cell magnetic correlations near optimal doping in $\mathrm{YBa}_{2} \mathrm{Cu}_{3} \mathrm{O}_{6.85}$. Nat. Commun. 6:7705 doi: 10.1038/ncomms8705 (2015).

(c) (i) This work is licensed under a Creative Commons Attribution 4.0 International License. The images or other third party material in this article are included in the article's Creative Commons license, unless indicated otherwise in the credit line; if the material is not included under the Creative Commons license, users will need to obtain permission from the license holder to reproduce the material. To view a copy of this license, visit http://creativecommons.org/licenses/by/4.0/ 